BULLETIN OF THE

AMERICAN MATHEMATICAL SOCIETY

Volume 80, Number 4, July 1974

\title{
A CLASS OF SQUARE-INTEGRABLE IRREDUCIBLE UNITARY REPRESENTATIONS OF SOME LINEAR GROUPS OVER COMMUTATIVE $p$-FIELDS ${ }^{1}$
}

\author{
BY R. G. LAHA
}

Communicated by François Treves, October 22, 1973

Let $k$ be a commutative $p$-field which is not necessarily of characteristic 0 (cf. [2]). We denote by $\mathcal{O}, \mathscr{P}$ and $\mathcal{O}^{\times}$the unique maximal compact subring of $k$, the unique maximal ideal contained in $\mathcal{O}$ and the group of invertible elements of the ring $\mathcal{O}$, respectively. Then the residue class field $\mathcal{O} / \mathscr{P}$ is a finite field of characteristic $p(p>1$ being a prime number). Let $n>1$ be a fixed positive integer. Let $G$ be the subgroup of $\operatorname{GL}(n, k)$ consisting of those elements whose determinant belongs to $\mathcal{O}^{\times}$. Then $K=\mathrm{GL}(n, \mathcal{O})$ is a maximal compact subgroup of $G$.

Recently Shintani [1] constructed some square integrable irreducible unitary representations of $G$ which are induced by suitable irreducible unitary representations of $K$, where these representations of $K$ can be 'parametrized' by certain characters of suitable compact Cartan subgroups of $G$ which are contained in $K$. However this construction is based on the assumption that $n$ and $p$ are relatively prime.

In the present note we show that an interesting subclass of these representations of $G$ can be constructed without this assumption. Moreover we give a more explicit description of the structure of these representations of $G$ in this case.

Formulation of the main result. Let $k$ be a commutative $p$-field. Let $\mathcal{O}, \mathscr{P}, \mathcal{O} \times$ be the maximal compact subring of $k$, the maximal ideal in $\mathcal{O}$ and the group of units in $\mathcal{O}$, respectively. Let $\pi$ be a prime element of $k$ and let $q$ be the module of $k$. Then the residue class field $\tilde{k}=\mathcal{O} \mid \mathscr{P}$ is a finite field of characteristic $p(p>1)$ containing $q$ elements. For every $v \in Z$, we write $\mathscr{P}^{v}=\pi^{v} \mathcal{O}$ where $\mathscr{P}^{0}=\mathcal{O}$. Let $n>1$. Let $G$ be the subgroup of the group $\mathrm{GL}(n, k)$ consisting of those elements whose determinant belongs to $\mathcal{O}^{\times}$. Let $K=\mathrm{GL}(n, \mathcal{O})$. Then $G$ is a unimodular locally compact topological group and $K$ is a maximal compact subgroup of $G$. We also note that the group $G$ is totally disconnected. For every positive integer $m \geqq 1$, we set $K_{m}=\left\{x \in K: x \equiv I_{n}\left(\bmod \mathscr{P}^{m}\right)\right\}$. Then $K_{m}$ is a compact open normal subgroup of finite index in $K$ and moreover $K_{1} \supset K_{2} \supset \cdots$ form a fundamental system of neighborhoods of the identity $I_{n}$ in $K$.

Let $k^{\prime}$ be an unramified extension of $k$ of degree $n$ over $k$. Let $\mathcal{O}^{\prime}$,

AMS (MOS) subject classifications (1970). Primary 22E55; Secondary 33A75.

1 This work was supported by National Science Foundation grant NSF-GP-35724 X. 
$\mathscr{P}^{\prime}$ and $\mathscr{O}^{\prime} \times$ be the maximal compact subring of $k^{\prime}$, the maximal ideal in $\mathcal{O}^{\prime}$ and the group of units in $\mathcal{O}^{\prime}$, respectively. Then we note that $k^{\prime}$ is generated over $k$ by a primitive $\left(q^{n}-1\right)$ th root of unity. Let $q^{\prime}=q^{n}$. Let $\zeta$ be a primitive $\left(q^{\prime}-1\right)$ th root of unity. Then $\zeta \in \mathcal{O}^{\prime} \times$ and moreover we have $\mathcal{O}^{\prime}=\mathcal{O}[\zeta]$ and $k^{\prime}=k(\zeta)$.

For every positive integer $m \geqq 1$, we define the subgroup $\mathscr{Q}_{m}^{\prime}$ of the group $\mathcal{O}^{\prime} \times$ by the formula $\mathscr{Q}_{m}^{\prime}=\left\{a \in \mathscr{O}^{\prime} \times: a-1 \in \mathscr{P}^{\prime m}\right\}$. Then $\mathscr{Q}_{m}^{\prime}$ is a compact open subgroup of finite index in $\mathcal{O}^{\prime} \times$ and moreover the subgroups $\mathscr{Q}_{1}^{\prime} \supset \mathscr{Q}_{2}^{\prime} \supset \cdots$ form a fundamental system of neighborhoods of the identity in $\mathcal{O}^{\prime} \times$.

Let $\omega$ be an arbitrary character of the multiplicative group $\mathcal{O}^{\prime} \times$ and let $\mathscr{P}^{\prime} r$ be the conductor of $\omega$. We assume $r \geqq 2$ and set $s=[r / 2]$. Let $\chi$ be a character of the additive group of $k$ of order 0 . Then there exists an element $x_{\omega} \in \mathcal{O}^{\prime}$ such that the relation

$$
\omega(a)=\chi\left(\operatorname{Tr}_{k^{\prime} / k}\left(\pi^{-r} x_{\omega}(a-1)\right)\right)
$$

holds for all $a \in \mathscr{Q}_{r-s}^{\prime}$. The character $\omega$ of $\mathcal{O}^{\prime} \times$ is said to be primitive if $x_{\omega}=\zeta$. Let $\omega$ be a primitive character of $\mathscr{O}^{\prime} \times$ and let $\mathscr{P}^{\prime} r$ be the conductor of $\omega$. Let $\tau$ be the embedding of $k^{\prime}$ into $\mathscr{M}(n, k)$ as defined in [1]. Then $A=\tau\left(\mathcal{O}^{\prime} \times\right)$ is a compact Cartan subgroup of $G$ such that $A \subset K$. We now define the character $\omega$ of the group $A$ by the formula $\omega(\tau(a))=\omega(a)$ for $a \in \mathcal{O}^{\prime} \times$.

We set $X=\tau(\zeta)$. Then $X \in \mathscr{M}(n, \mathcal{O})$. Then we define the one-dimensional representation $\chi_{X}^{s}$ on $K_{r-s}$ by the formula

$$
\chi_{X}^{r}(x)=\chi\left(\pi^{-r}(\operatorname{Tr} X(x-1))\right), \quad x \in k_{r-s} .
$$

Let $K_{X}$ be the centralizer of $\chi_{X}^{r}$ in $K$. We now assume that $r$ is even so $r=2 s$ and hence $r-s=s$. Then $K_{X}=A K_{s}$. We now define the function $\lambda_{\omega}$ on $K_{X}$ by the formula

$$
\lambda_{\omega}(a x)=\omega(a) \chi_{X}^{2 s}(x), \quad a \in A, x \in K_{s} .
$$

Then $\lambda_{\omega}$ is a one-dimensional representation of $K_{X}$ which coincides with $\omega$ on $A$ and with $\chi_{X}^{2 s} \cdot 1$ on $K_{s}=K_{r-s}$.

Then the main result can be formulated as follows.

THEOREM. (i) Let $\sigma_{\omega}=\operatorname{Ind}_{K_{\chi} \uparrow K} \lambda_{\omega}$. Then $\sigma_{\omega}$ is an irreducible unitary representation of $K$.

(ii) Let $\pi_{\sigma_{\omega}}=\operatorname{Ind}_{K \uparrow G} \sigma_{\omega}$. Then $\pi_{\sigma_{\omega}}$ is a square integrable irreducible unitary representation of $G$.

\section{REFERENCES}

1. T. Shintani, On certain square-integrable irreducible unitary representations of some P-adic linear groups, J. Math. Soc. Japan 20 (1968), 522-565. MR 38 \#2252.

2. A. Weil, Basic number theory, Die Grundlehren der math. Wissenschaften, Band 144, Springer-Verlag, New York, 1967. MR 38 \#3244.

Department of Mathematics, Bowling Green State University, Bowling GREEN, OHIO 43403 